\title{
Clinical Relevance of Corrosion Patterns Attributed to Inflammatory Cell Induced Corrosion: A Retrieval Study
}

Anna Di Laura ${ }^{1}$, Harry S. Hothi ${ }^{1}$, Jay Meswania ${ }^{1}$, Robert K Whittaker ${ }^{1}$, Danielle de Villiers ${ }^{1}$, Gordon W. Blunn ${ }^{\text {, John A. Skinner }}{ }^{1}$, Alister J. Hart ${ }^{1}$

1. Institute of Orthopaedics and Musculoskeletal Science, University College London and the Royal National Orthopaedic Hospital, Stanmore, United Kingdom

Corresponding Author:

Anna Di Laura

Institute of Orthopaedics and Musculoskeletal Science (University College London)

Royal National Orthopaedic Hospital

Brockley Hill

Stanmore, Middlesex

HA7 4LP

United Kingdom

Phone: +44 (0) 2089095825

Fax: $\quad+44(0) 2089548560$

Email: anna.laura.14@ucl.ac.uk 


\begin{abstract}
In vitro studies have shown that human osteoclasts can corrode stainless steel and titanium leading to the production of metal ions responsible for inflammatory reactions. Moreover, traces of cellular activities on metal orthopaedic explants have recently been reported as Inflammatory Cell-Induced (ICI) corrosion being the result of the cells sealing on the metal surfaces and releasing Reactive Oxygen Species (ROS) through Fenton-like reactions. The extent and clinical relevance of this phenomenon has yet to be understood. We analysed a cohort of $100 \mathrm{CoCr}$ alloy hips collected at our retrieval centre; we performed macroscopic and microscopic screening and used statistical analysis to correlate our findings with implant and clinical variables. We found that $59 \%$ of our implants had evidence of surface damage consistent with what has previously been described as cell-induced corrosion. There was a significant association between the patterns and aseptic loosening for the ASR modular ( $\mathrm{r}=-$ 0.488, $\mathrm{p}=0.016)$ and the Durom modular $(\mathrm{r}=0.454, \mathrm{p}=0.026)$. This is the largest implant retrieval study to examine the phenomena of so-called ICI corrosion and is the first to investigate its clinical relevance. We recommend further work to determine the role of cells in the damage patterns observed.
\end{abstract}

Keywords: Corrosion; Cell; CoCr; Inflammation; Orthopaedic Implants 


\section{Introduction}

Cobalt Chromium (CoCr) alloys are the most commonly used materials in orthopaedics, due to their favourable mechanical and tribological properties (1). However, there are concerns about their corrosion and subsequent release of both soluble and particulate implant debris in vivo that leads to inflammatory/immune responses (2-6).

To date, studies in vitro have demonstrated that human osteoclast precursors can attach, grow and differentiate towards mature osteoclasts on titanium and stainless steel and corrode the surface, leading to the formation of metal ions that may cause immune and/or inflammatory reaction $(7,8)$. Monocytes differentiated into osteoclasts have been added stainless steel discs and shown to cause resorption pits (8). Moreover, evidence of direct Inflammatory Cellinduced Corrosion (ICI) on CoCr implants has been demonstrated by a retrieval study conducted by Gilbert et al. (9) in which the morphology of the corrosion patterns, size as well as the presence of cellular remnants attached to the surface corresponded to the actual features of attaching inflammatory cells.

The cellular attack itself seems to be a new in vitro recognised phenomenon and it may represent a specific mechanism of metal corrosion occurring in vivo contributing to the rise of metal ions levels as well as implant failure. This may suggest a mechanism of failure reported as unexplained pain in well-positioned implants thought to be the result of adverse tissue reactions (10), or osteolysis with consequent aseptic loosening of the implants associated with excessive immune reaction to metal particles released into the body. In this respect, it has been shown that $\mathrm{CoCr}$ particles can trigger the release of pro inflammatory cytokines by monocytes as well as macrophages, eliciting osteoclastogenesis and resorption activity (2).

The extent and clinical relevance of cell-induced corrosion is not clear. We aimed to better understand the clinical relevance of this phenomenon. Our objectives were 1) to determine the extent of ICI corrosion patterns observed in a large cohort of retrieved hip implants, 2) to give 
a 3-dimensional characterisation to the corroded regions, 3) to correlate our findings with clinical data and 4) to predict which patients are more likely to be affected.

\section{Materials and Methods}

This was a retrieval study involving 100 failed hip implants received at our centre with metalon-metal (MOM) bearings. We selected Durom (Zimmer, Winterthur, Switzerland) modular heads [ $\mathrm{n}=25]$, Durom resurfacing $[\mathrm{n}=25]$ as well as ASR (DePuy Orthopaedics, Leeds, UK) modular heads [ $n=25]$ and ASR resurfacing [n=25] for inspection, so that we included both metallurgical features for Co-Cr alloys: wrought and cast. For each implant, we also collected clinical information inclusive of gender, age, reason for revision, cobalt and chromium blood levels and time of implantation. Retrievals were obtained from 61 female and 39 male patients with a median age of 57 (23-79) years; the median time to revision was 37 (15-129) months. The median concentration of cobalt in the whole blood was $6.59(0.5-163)$ ppb whereas for chromium it was $3.54(0.2-119) \mathrm{ppb}$. The most prevalent reasons for revision were unexplained pain $(n=55)$, aseptic loosening $(n=24)$, infection $(n=6)$, ALVAL $(n=5)$, periprosthetic fracture $(n=3)$, pseudotumors $(n=2)$, one case of malalignment and for 4 explants the reason was not reported.

We investigated the presence of specific patterns described by Gilbert et al. (9). Discoloured or light frosted areas macro and microscopically visible that appeared to consist of streaks of pits, haziness or crater-like features at higher magnifications and/or scanning electron microscopy. We noticed presence of cellular remnants on the implant surfaces and iron accumulation in correspondence of areas of corrosion.

The components were studied according to a published method (11) dividing them into polar and equatorial regions (Figure 1). 
Moreover, a photogrammetric method to measure the area of damage was implemented using an imaging system (EOS 5D Mark II camera, Canon, Tokyo, Japan) and public-domain software for image analysis (Image J 1.4.3.67, Broken Symmetry Software). Image calibration was achieved by positioning a reference scale in the field of view.

\section{Implant preparation}

In order to eliminate the possibility that the patterns were due to biological remnants on the surface, three cleaning methods reported in the literature were tested on some of the implants with no changes as a result. In detail, the devices were: 1) soaked in $10 \%$ solution of Decon 90 in an ultrasonic cleaning bath for 30 minutes, subsequently rinsed in distilled water and left to dry in air for 1 hour $(12,13) ; 2)$ cleaned in repeated ultrasonic baths of acetone, methanol, distilled water, and again acetone (14); 3) soaked in a $5 \mathrm{~mol} / \mathrm{l}$ and $10 \mathrm{~mol} / \mathrm{l}$ sodium hydroxide for 48 hours, then rinsed in deionised water and allowed to dry $(15,16)$.

\section{Visual assessment}

Initial examination included macroscopic and microscopic analysis of both cups and heads with the aid of a Leica M50 (Leica Microsystems, Germany) stereomicroscope and a Keyence VHX-700F series (Keyence Co., Japan) digital microscope to assess and record the presence and localisation of patterns on the surfaces with magnification ranging from $5 \mathrm{x}$ up to $1000 \mathrm{x}$.

\section{Scanning Electron Microscopy and Energy Dispersive X-ray Spectrometry}

A Scanning Electron Microscope SEM (Jeol JSM5500, Tokyo, Japan) was used to perform detailed microscopic analysis of specific areas of interest highlighted from the initial visual inspection. Second Electron Imaging (SEI) ranging from low (4-5) to high (10-15) kV and high $\mathrm{kV}$ (10-15) Backscattered imaging (BEC) were used. Low $\mathrm{kV}$ imaging of non-conducting 
material does not penetrate cell membranes and can reveal biological materials. High energy electron beams SEI gives topographic information of the alloy itself, while high voltage BEC provides both topographic and compositional information of the metal surface. Energy dispersive X-ray spectrometry (EDS) was performed for different regions on the component to assess the elemental composition and organic biocorrosion products on the implant surface.

\section{Optical Profilometry}

We used a 3D optical microscope (Bruker, ContourGT inMotion, Massachusetts, USA) to identify features of corrosive damage. Generated area topography maps with a Gaussian regression filter showed both the depth of pits and deposition of material in $\mu \mathrm{m}$.

\section{Statistical Analysis}

The significance of any univariable associations between the presence of ICI corrosion as well as area of damage and key implant and clinical variables were assessed using simple linear regression models. All statistical analysis was performed using Stata/IC version 12.1 (StataCorp, College Station, TX, USA) and throughout a $\mathrm{p}$ value $<0.05$ was considered significant.

\section{Results}

Our screening revealed that 59\% of implants (60\% ASR modular, 44\% ASR resurfacing, 68\% Durom modular, 64\% Durom resurfacing) had evidence of corrosion patterns described by Gilbert et al. (9), all of them localised in the equatorial zones of the components, thus away from the contact regions. Haziness pits and streaks were visible on the polished regions [Figure2 (A) and Figure 6 (A)] of the heads as well as on internal edges of cups [Figure 4 (A)], on the machining marks of acetabular rims [(Figure $5(\mathrm{~A})]$ and head rims [(Figure $3(\mathrm{~A})]$. Higher 
magnification images showed crater-like spots surrounded by a frosted pattern of a ruffled topography [Figure 5-6 (B-C)] or circular rings attributed to moving cells [Figure 4 (B-C)]. Machining lines were damaged by ICI corrosion in the same way as polished areas (Figure 3).

\section{SEM Analysis}

SEM analysis confirmed the presence of the unique features derived from different cellular species and/or activities (Figure 7). Figure 8 shows low voltage SEI (A), high voltage SEI (B) and high voltage BEC (C) images detailing biological material (bright spot in A) in the corroded region and the metal surface of the alloy underneath the biological residue $(\mathrm{B}, \mathrm{C})$. EDS analysis revealed the presence of iron in correspondence of corroded regions (Figure 9), Iron was only detectable in proximity of the corrosion patterns.

\section{Optical Profilometry}

Crater-like features revealed under microscopic (both optical and scanning electron) analysis were seen under the optical profilometer (Figure 10-11). Pits or excavations surrounded by round depositions were common findings with depositions appearing to be of bigger area. This may be due to the white-light measurements reflecting off biological remnants within the corroded regions. Another plausible reason is the electrodeposition of metal (much like the iron streaks) that could include Co and Cr ions due to the complex redox interactions between ROS and $\mathrm{CoCr}$ in the presence of active cell attack; thus, metal dissolves (oxidizes) and deposits (reduces) within the surface-cell space. It may also be due to oxidation processes generating larger oxide deposits in the presence of oxygen and/ or at different $\mathrm{pH}$. Scaled maps gave information about depth of pits that ranged from 0.5 to $3 \mu \mathrm{m}$ [Figure 11)]. 


\section{Statistical Analysis}

We did not find any significant associations between the presence of ICI corrosion and time to revision, taper corrosion score, gender, head size, age or metal ion levels for all four implant types, Table 1 . We found that there was a significant association between ICI corrosion and aseptic loosening for the ASR modular $(\mathrm{r}=-0.488, \mathrm{p}=0.016)$ and the Durom modular $(\mathrm{r}=0.454$, $\mathrm{p}=0.026$ ) hips. There was no significant association between ICI corrosion and unexplained pain for any of the four implant types.

Furthermore, we did not find significant association between the area of corrosion damage and the implants failure due to aseptic loosening $(\mathrm{n}=24)$ and unexplained pain $(\mathrm{n}=55)$. The median corrosion area for loose implants was $8.4 \mathrm{~mm}^{2}(2.4-21.6)$, and for non-loose implants was $8.7 \mathrm{~mm} 2(2.8-34.0), \mathrm{p}=0.8312$. The median corrosion area for implants failed due to unexplained pain was $9.9 \mathrm{~mm}^{2}(2.8-34.0)$, for those failed for other reasons was $7.8 \mathrm{~mm}^{2}(2.4$ $-27.8), p=0.3714$.

\section{Discussion}

Our findings support previously reported observations (9), about evidence of possible cell induced corrosion. To further show evidence, we eliminated possible bias associated with biological deposition on the metal alloys of the explants and characterised the features. We did not find any significant associations between the unique features of corrosion and the implant design or metallurgy meaning that the phenomenon might be widely present. Moreover, we analysed the correlation between its presence and the reasons for revision, time to revision, Goldberg score (for modular heads), gender, head size, Co level, $\mathrm{Cr}$ level and age; we found a link between the ICI patterns and aseptic loosening of modular head hip implants. 
Despite technological advancements in providing biocompatible metal alloys for orthopaedic implants, their susceptibility to undergo corrosion releasing ions and particles in vivo remains a concern (17). The release of cobalt and chromium from the bearing surfaces and taper junctions can induce inflammatory reactions mediated by resident and recruited cells $(6,18)$ leading to implant failure $(19,20)$.

The nanoparticles generated in metal-on-metal prosthesis can dissolve into local fluid, penetrate cells, remain around the joint or enter the systemic circulation being responsible for evoking adverse physiological effects including metal sensitivity and cytotoxicity $(21,22)$. The presence of wear particles from Co-Cr alloys into the periprosthetic tissue elicits the activity of immune cells which release inflammatory cytokines such as interleukin IL-1 $\alpha / 1 \beta$, IL-6 and tumor necrosis factor TNF- $\alpha$ leading to an increased osteolytic activity $(2,22)$. Several studies have reported the effects of metal exposure on the host environment concluding that the toxicity depends on particle size, shape and concentration $(22,23)$. The wear volume generated by metal-on-metal articulations is 40-100 times lower than those produced by metal-onpolyethylene bearings, with this not being indicative of poorer biological impact (24). In fact, despite the difference in volume, the particles range in size from 51 to $116 \mathrm{~nm}(25)$, significantly smaller than polyethylene particles and therefore the number of particles exceeds that produced in metal-on-polyethylene articulations (25).

Moreover, it has been showed that the risk of revision surgeries is positively linked with the levels of $\mathrm{Co}$ and $\mathrm{Cr}$ in the blood (26). Specificity on Cobalt and Chromium release seems to be in existence, with Co being more reactive and soluble, thus more important in the inflammatory process that $\mathrm{Cr}(26,27)$. In detail, it has been found that, whereas high chromium concentrations induce cell death by apoptosis, high cobalt concentrations produce cell necrosis with consequent strong inflammatory reaction of the surrounding tissue (27). Therefore, the amount of particles/ions that accumulates around the implant is responsible for the creation of 
a chronic inflammation leading to a decreased level of oxygen, also called hypoxia. Another factor that contributes to the hypoxia around a corroding implant is the associated reduction reactions occurring elsewhere on the implant due to a negative shift in potential of the whole implant, these reduction reactions may locally deplete the concentration of solution-dissolved oxygen and affect cell behaviour in the periprosthetic environment (28). It has been shown that a reduced oxygen tension can elicit the formation and/or activation of the monocytemacrophage lineage cells. In particular, it stimulates osteoclastogenesis and its activity together with both increasing the size of osteoclasts and the area of resorption pits compared to nonhypoxic conditions. This is also linked with the fact that hypoxic tissues become acidified thus worsening the resorptive activity of osteoclasts known to be dependent on the $\mathrm{pH}$. These cells, as well as macrophages, neutrophils and chondrocytes, in particular conditions, can switch their metabolism being able to rely on glycolysis to survive (29). In addition, it has been studied that hypoxia also exerts an effect on osteoblasts by limiting their proliferation and maturation, therefore enhancing bone resorption (30).

Innate and adaptive responses to wear and corrosion products comprise a variety of cells. Phagocytic cells involved in inflammatory processes include macrophages, osteoclasts, and giant cells whereas immune cells involved are mainly lymphocytes, neutrophils and plasma cells (6). Osteoclasts are mono or multinucleated cells deriving from marrow-derived mononuclear precursors that circulate in the monocyte fraction activated in the presence of macrophage-colony stimulating factor (M-CSF) and the receptor activator of NF-KB ligand (RANK-L) expressed by osteoblasts and stromal cells $(7,8,31)$. They are highly-specialised bone-resorbing cells characterised by a basal ruffled border that creates a seal around the site of attack forming an acidic environment ( $\mathrm{pH}$ value around 4.5) by activation of proton pumps present in the membrane of the ruffled border $(7,32)$. The hypothesis that cells seal on the surface alloy and can directly create an acidic and concentrated ROS environment so as to 
destabilise the passivation layer and make the metal prone to dissolve is a new concept. The caused damage may not be quantitatively substantial compared to the corrosion mechanisms occurring at the modular junctions but may elicit a reaction just as comparable such as to lead to implant failure. In the ruffled border of phagocytic cells, Fenton reactions take place. Reactive Oxygen Species are the principal mediators in the defence against bacteria or foreign bodies as well as in bone remodelling (33). ROS like hydrogen peroxide $\mathrm{H}_{2} \mathrm{O}_{2}$ and superoxide $\mathrm{O}_{2}{ }^{-}$are essential to life, being involved in many different biological functions. When overproduced, they become highly harmful, causing oxidative stress through the oxidation of biomolecules leading to locally severe inflammatory processes (34). It has been demonstrated that hydrogen peroxide increases the corrosion susceptibility of $\mathrm{CoCr}$ alloys (9). In this way, the metal is more prone to be corroded due to the destabilised passivation layer and due to the presence of metal ions on the surface leading to additional exposure for corrosion (7); the release of metal ions further worsens the production of free radicals (33).

Iron is a fundamental component of phagocytic cells and it is known to exacerbate inflammation in the presence of ROS. Via the Fenton reaction, the $\mathrm{O}_{2}{ }^{-}$can mobilise Fe from intracellular storage ferritin, this $\mathrm{Fe}$ can then form toxic species like hydroxyl radical $\mathrm{OH}^{\prime}$ and hydrogen peroxide (34) thus perpetuating the inflammatory cascade.

In this study, the morphology of the patterns are consistent with previous in vitro findings in which phagocytic cells attached the metal surface and created lacunar resorption sites on stainless steel and titanium alloys $(7,8)$; in addition, the patterns are consistent with other studies where the osteoclasts were cultured on dentin, calcium phosphate (35) and cortical bone (31). Presence of migratory cells [Figure 4(B-C)] that form streaks of corrosion (Figure 2-3-6) can be indicative of cell motion following specific tribological or chemotactic stimuli as wear particles from the bearing surfaces or from head-stem junction. 
The results show no difference in the percentage of presence between cast and wrought microstructures in accordance with Gilbert's findings. In addition, there is evidence of correlation between the implants revised due to aseptic loosening and presence of cellular corrosion in the case of modular heads hips. Iron is also in accordance with the presence of phagocytic cells on the metal surfaces due to Fenton-like reaction taking place.

All these mechanisms are linked with the pathophysiological process of bone homeostasis leading to unpaired resorption, immune reactivity and severe inflammatory reactions and may partially explain the phenomenon of aseptic loosening and implant failure (20). However, we have yet to identify clinical predictors for this in vivo mechanism despite the analysis of clinical data for 100 cases with the majority failed for unexplained pain. Additional work is required to identify the precise role of cellular attack in the corrosion damage patterns observed and furthermore the cell types involved are unclear.

\section{Acknowledgements}

We are grateful for the support of Gwynneth Lloyd and Elizabeth Ellis for their coordination of the retrieval centre. Additionally we thank Professor Jeremy Gilbert for his valuable discussions during this study.

Two authors received funding from the British Orthopaedic Association through an industry consortium of nine manufacturers: DePuy International Ltd (Leeds, UK), Zimmer GmbH (Winterthur, Switzerland), Smith \& Nephew UK Ltd (Warwick, UK), Biomet UK Ltd (Bridgend, South Wales, UK), JRI Ltd (London, UK), Finsbury Orthopaedics Ltd (Leatherhead, UK), Corin Group PLC (Cirencester, UK), Mathys Orthopaedics Ltd (Alton, UK), and Stryker UK Ltd (Newbury, UK). 


\section{References}

1. Schröck K, Lutz J, Mändl S, Hacker MC, Kamprad M, Schulz-Siegmund M. Co (II)mediated effects of plain and plasma immersion ion implanted cobalt-chromium alloys on the osteogenic differentiation of human mesenchymal stem cells. Journal of Orthopaedic Research. 2014.

2. Caicedo MS, Pennekamp PH, McAllister K, Jacobs JJ, Hallab NJ. Soluble ions more than particulate cobalt-alloy implant debris induce monocyte costimulatory molecule expression and release of proinflammatory cytokines critical to metal-induced lymphocyte reactivity. Journal of Biomedical Materials Research Part A. 2010;93(4):1312-21.

3. Lee SH, Brennan F, Jacobs J, Urban R, Ragasa D, Glant T. Human monocyte/macrophage response to cobalt-chromium corrosion products and titanium particles in patients with total joint replacements. Journal of orthopaedic research. 1997;15(1):40-9.

4. Wooley P, Schwarz E. Aseptic loosening. Gene therapy. 2004;11(4):402-7.

5. Lassus J, Salo J, Jiranek WA, Santavirta S, Nevalainen J, Matucci-Cerinic M, et al. Macrophage activation results in bone resorption. Clinical orthopaedics and related research. 1998;352:7-15.

6. Bauer TW, Campbell PA, Hallerberg G, Group BW. How have new bearing surfaces altered the local biological reactions to byproducts of wear and modularity? Clinical Orthopaedics and Related Research®. 2014;472(12):3687-98.

7. Cadosch D, Al-Mushaiqri MS, Gautschi OP, Meagher J, Simmen HP, Filgueira L. Biocorrosion and uptake of titanium by human osteoclasts. Journal of Biomedical Materials Research Part A. 2010;95(4):1004-10.

8. Cadosch D, Chan E, Gautschi OP, Simmen HP, Filgueira L. Bio-corrosion of stainless steel by osteoclasts - in vitro evidence. Journal of Orthopaedic Research. 2009;27(7):841-6.

9. Gilbert JL, Sivan S, Liu Y, Kocagöz SB, Arnholt CM, Kurtz SM. Direct in vivo inflammatory cell-induced corrosion of CoCrMo alloy orthopedic implant surfaces. Journal of Biomedical Materials Research Part A. 2014.

10. Hart AJ, Matthies A, Henckel J, Ilo K, Skinner J, Noble PC. Understanding Why Metalon-Metal Hip Arthroplasties FailA Comparison Between Patients with Well-Functioning and Revised Birmingham Hip Resurfacing Arthroplasties AAOS Exhibit Selection. The Journal of Bone \& Joint Surgery. 2012;94(4):e22 1-10.

11. Hothi HS, Berber R, Whittaker RK, Bills PJ, Skinner JA, Hart AJ. Detailed inspection of metal implants. Hip international: the journal of clinical and experimental research on hip pathology and therapy. 2015:0-.

12. Flannery M, Jones E, Birkinshaw C. Compliant layer knee bearings. Part II: Preliminary wear assessment. Wear. 2010;269(5):331-8.

13. Lee JK, Maruthainar K, Wardle N, Haddad F, Blunn GW. Increased force simulator wear testing of a zirconium oxide total knee arthroplasty. The Knee. 2009;16(4):269-74.

14. Böhler M, Kanz F, Schwarz B, Steffan I, Walter A, Plenk H, et al. Adverse tissue reactions to wear particles from Co-alloy articulations, increased by alumina-blasting particle contamination from cementless Ti-based total hip implants a report of seven revisions with early failure. Journal of Bone \& Joint Surgery, British Volume. 2002;84(1):128-36.

15. Minoda Y, Kobayashi A, Iwaki H, Miyaguchi M, Kadoya Y, Ohashi H, et al. Characteristics of polyethylene wear particles isolated from synovial fluid after mobile-bearing and posterior-stabilized total knee arthroplasties. Journal of Biomedical Materials Research Part B: Applied Biomaterials. 2004;71(1):1-6.

16. Campbell P, Ma S, Schmalzried T, Amstutz HC. Tissue digestion for wear debris particle isolation. Journal of biomedical materials research. 1994;28(4):523-6. 
17. Jacobs JJ, Hallab NJ, Skipor AK, Urban RM. Metal degradation products: a cause for concern in metal-metal bearings? Clinical orthopaedics and related research. 2003;417:139-47. 18. Caicedo MS, Samelko L, McAllister K, Jacobs JJ, Hallab NJ. Increasing both CoCrMoalloy particle size and surface irregularity induces increased macrophage inflammasome activation in vitro potentially through lysosomal destabilization mechanisms. Journal of Orthopaedic Research. 2013;31(10):1633-42.

19. Jacobs JJ, Hallab NJ. Loosening and osteolysis associated with metal-on-metal bearings: a local effect of metal hypersensitivity? The Journal of Bone \& Joint Surgery. 2006;88(6):11712.

20. Goodman SB. Wear particles, periprosthetic osteolysis and the immune system. Biomaterials. 2007;28(34):5044-8.

21. Bradberry S, Wilkinson J, Ferner R. Systemic toxicity related to metal hip prostheses. Clinical Toxicology. 2014;52(8):837-47.

22. Sommer B, Felix R, Sprecher C, Leunig M, Ganz R, Hofstetter W. Wear particles and surface topographies are modulators of osteoclastogenesis in vitro. Journal of Biomedical Materials Research Part A. 2005;72(1):67-76.

23. Polyzois I, Nikolopoulos D, Michos I, Patsouris E, Theocharis S. Local and systemic toxicity of nanoscale debris particles in total hip arthroplasty. Journal of Applied Toxicology. 2012;32(4):255-69.

24. Ingham E, Fisher J. Biological reactions to wear debris in total joint replacement. Proceedings of the Institution of Mechanical Engineers, Part H: Journal of Engineering in Medicine. 2000;214(1):21-37.

25. Doorn PF, Campbell PA, Worrall J, Benya PD, McKellop HA, Amstutz HC. Metal wear particle characterization from metal on metal total hip replacements: transmission electron microscopy study of periprosthetic tissues and isolated particles. Journal of biomedical materials research. 1998;42(1):103-11.

26. Hart AJ, Quinn PD, Lali F, Sampson B, Skinner JA, Powell JJ, et al. Cobalt from metalon-metal hip replacements may be the clinically relevant active agent responsible for periprosthetic tissue reactions. Acta biomaterialia. 2012;8(10):3865-73.

27. Granchi D, Cenni E, Ciapetti G, Savarino L, Stea S, Gamberini S, et al. Cell death induced by metal ions: necrosis or apoptosis? Journal of materials science: materials in medicine. 1998;9(1):31-7.

28. Gilbert JL, Zarka L, Chang E, Thomas $\mathrm{CH}$. The reduction half cell in biomaterials corrosion: oxygen diffusion profiles near and cell response to polarized titanium surfaces. Journal of biomedical materials research. 1998;42(2):321-30.

29. Utting JC, Flanagan AM, Brandao-Burch A, Orriss IR, Arnett TR. Hypoxia stimulates osteoclast formation from human peripheral blood. Cell biochemistry and function. 2010;28(5):374-80.

30. Utting J, Robins S, Brandao-Burch A, Orriss I, Behar J, Arnett T. Hypoxia inhibits the growth, differentiation and bone-forming capacity of rat osteoblasts. Experimental cell research. 2006;312(10):1693-702.

31. Neale SD, Haynes DR, Howie DW, Murray DW, Athanasou NA. The effect of particle phagocytosis and metallic wear particles on osteoclast formation and bone resorption in vitro. The Journal of arthroplasty. 2000;15(5):654-62.

32. Arnett TR, Dempster DW. Effect of $\mathrm{pH}$ on bone resorption by rat osteoclasts in vitro. Endocrinology. 1986;119(1):119-24.

33. Rosen GM, Pou S, Ramos CL, Cohen MS, Britigan BE. Free radicals and phagocytic cells. The FASEB Journal. 1995;9(2):200-9.

34. Blake DR, Merry P, Stevens C, Dabbagh A, Sahinoglu T, Allen R, et al. Iron free radicals and arthritis. Proceedings of the Nutrition Society. 1990;49(02):239-45. 
35. Schilling AF, Linhart W, Filke S, Gebauer M, Schinke T, Rueger JM, et al. Resorbability of bone substitute biomaterials by human osteoclasts. Biomaterials. 2004;25(18):3963-72.

\section{Figure Legends}

Figure 1: Schematic diagram showing the method used to localise corrosion damage on the components. a) Cup bearing surface considered in terms of 8 zones: 4 zones in the polar (P) regions and 4 zones in the equatorial (E) regions; b) head bearing surface considered in terms of 8 zones: 4 zones in the polar $(\mathrm{P})$ regions and 4 zones in the equatorial $(\mathrm{E})$ regions. (Taken from Hothi et al., 2014).

Figure 2: Low magnification DOM images of: (A) ASR modular (B) ASR resurfacing, (C) Durom modular and (D) Durom resurfacing heads showing location and type of damage (arrows). Streaks and haziness are identifiable on the polished surface of the components mainly at the level of the equator in the non-contact region. Streaks suggestive of cell motility presumably attracted by tribological stimuli or wear ions/particulate from the bearing surfaces.

Figure 3: DOM images at different magnifications of a streak of corrosion on the rim of a CoCr head in proximity of the modular taper bore. In image (C) it is possible to distinguish the damage caused by the corrosion on the machining lines.

Figure 4: Images showing streaks of corrosion on the internal surface and on the edge of the acetabular cup (A and B). In (C) a particular pattern of image (B) has been magnified (arrows) showing a crater like shape surrounded by concentric rings of about $100 \mu \mathrm{m}$ in diameter. Size of patterns on our collections ranged between 10 to $200 \mu \mathrm{m}$. 
Figure 5: Series of images showing damage on the rim of a metal acetabular component (arrow). 4 (B) and (C) show haziness and discoloured areas at higher magnifications.

Figure 6: Micrograph showing isolated crater-like patterns on a $\mathrm{CoCr}$ head [Figure 1 (C)]. Frosted patterns of ruffled topography surrounding circular spots were common findings in our studied collection.

Figure 7: SEI secondary mode images of the modular head in Figure 6 showing different patterns occurring on the same component, presumably due to different cell types and/or activities. Detail of the strike of corrosion (A, B) surrounded by undamaged surface. Image $(C, D)$ show isolated spots of corrosion of a larger diameter. $(E)$ and $(F)$ document the topography of ruffled borders typical of inflammatory cells.

Figure 8: Low voltage SEI (A), high voltage SEI (B) and high voltage BEC (C) images detailing biological material (bright spot in A) in the corroded region and the metal surface of the alloy underneath the biological residue $(\mathrm{B}, \mathrm{C})$.

Figure 9: EDS reveals presence of Fe presumably deriving from Feanton-like reactions involving reactive oxygen species.

Figure 10: Surface maps of a region that appeared hazy showed in Figure 1B, crater-like patterns revealed under a 3D optical microscope. 
Figure 11: Particular point (blue area) on the map evaluated per depth. The graphs on the right show a depth of about $1 \mu \mathrm{m}$ (red arrows). Central corrosion damage (blue spots), with edge deposition (around $1 \mu \mathrm{m}, \mathrm{Y}$ profile), visible on both big and small crater-like patterns. 